\title{
Gravitino Dark Matter Candidate and Big Bang Nucleosynthesis
}

\author{
Sean BAILLY*i \\ LAPTH \\ E-mail: sean.bailly@lapp.in2p3.fr
}

\begin{abstract}
The gravitino as the lightest supersymmetric particle has proven to be an interesting candidate for dark matter. It is mainly produced during reheating after inflation and from the decay of the next-to-lightest supersymmetric particle (NLSP). Scenarios with a stau NLSP can also reconcile observationally inferred and theoretically predicted lithium abundances produced in Big Bang Nucleosynthesis. These scenarios require fairly massive supersymmetric particles and an upper bound on the reheating temperature too low to satisfy the requirements from thermal leptogenesis. We study such scenarios in the context of a non-standard cosmological evolution with a modified pre-BBN expansion rate. The reheating temperature bound can be strongly relaxed and the lithium problem solved with a lighter stau enabling possible production and detection at the LHC.
\end{abstract}

Identification of Dark Matter 2010

July 26 - 302010

University of Montpellier 2, Montpellier, France

* Speaker.

${ }^{\dagger}$ A footnote may follow. 


\section{Introduction}

Supersymmetric models with the gravitino as the lightest supersymmetric particle (LSP) with R-parity conservation have very interesting phenomenology despite the fact that they produce no direct and indirect detection signals. The gravitino is a candidate for dark matter and can be produced in many ways: from scattering processes during reheating or from the decay of the next-to-lightest supersymmetric particle (NLSP). The lifetime of the NLSP can be quite long (above one second) because its couplings to the gravitino are suppressed by the Planck mass. Consequently the decay of the NLSP to the gravitino along with Standard Model (SM) particles can occur during Big Bang Nucleosynthesis (BBN). The injection of SM particles induces electromagnetic or hadronic cascades that can alter the production of the light elements during BBN. Observations put strong constraints on the abundance of these elements and induce constraints on the characteristics of the NLSP: its abundance, lifetime, mass and decay branching ratios.

The standard picture of BBN (SBBN) assumes that there is no decay of an unstable relic particle occuring in the plasma at the time of BBN. The model depends on a single parameter, the baryon to photon ratio well measured by the WMAP mission [1] :

$$
\eta_{10}=10^{10} \frac{n_{b}}{n_{\gamma}}=6.225 \pm 0.170
$$

Predictions are in good agreement for deuterium and helium-4. Therefore SBBN is a rather good description of Big Bang Nucleosynthesis physics. For lithium-7, observations made in low metallicity regions present lower abundances by a factor a few compared to the theoretical model. In the 1980', measurements showed that lithium-7 abundance was independent of the metallicity for the low metallicity stars (Spite plateau). This was interpreted as the primordial abundance of lithium7 and therefore presented a discrepancy with the model. The origin of the problem could arise on the one hand from observation difficulties, incomplete modelisation of star mechanisms with turbulence and mixing, or on the other hand from the model with uncertainties on some reaction rates or an incomplete picture with the decay of unstable particles during BBN. Although refined observations of the Spite plateau show a little scattering and a double plateau making interpretation much more difficult, the lithium-7 problem still needs to be adressed. The lithium- 6 is much more difficult to measure than the lithium-7 but careful studies seem to point out to a lithium- 6 problem, observations of lithium- 6 abundance being a few orders of magnitude higher than the standard picture prediction.

To solve both lithium problems a possible scenario is to suppose the decay of the NLSP during BBN into the gravitino. The decay cascades would modify the abundance of lithium- 6 and lithium-7 without changing deuterium or helium-4. This was done in many studies, first with electromagnetic and hadronic decay and then by adding catalytic effects when the charged NLSP forms a bound state with the light elements. The scenario with a stau NLSP decaying to a gravitino can solve both lithium problems and it was shown that it was possible to account for the right amount of dark matter from the gravitino LSP. However this scenario requires a gravitino mass of $m_{3 / 2} \sim 100 \mathrm{GeV}$, a stau mass $m_{\tilde{\tau}} \sim 1.5 \mathrm{TeV}$ and a low reheating temperature $T_{R} \sim 10^{7} \mathrm{GeV}[2,3]$ well below temperature needed for a successful thermal leptogenesis scenario $\left(T_{R} \sim 10^{9} \mathrm{GeV}\right)$.

To reconcile the stau-gravitino scenario with thermal leptogenesis, one can assume a nonstandard cosmological history with for instance the presence of an additional energy density before 
the beginning of BBN which then becomes negligible in order not to interfere with observations. The model used in this study is presented in [4] and results are developed in [5]. Here we will first present this non-standard cosmological scenario and its implication on the expansion rate, then the consequences for the gravitino dark matter relic density and finally the stau decay to solve the BBN problems.

\section{Pre-BBN modified expansion}

In [4], an additional dark component with a positive energy density is added in the early universe, it is parametrized as follow :

$$
\rho_{D}(T)=\kappa_{D} \rho_{\mathrm{rad}}\left(T_{\mathrm{BBN}}\right)\left(\frac{T}{T_{\mathrm{BBN}}}\right)^{n_{D}}
$$

with $\kappa_{D} \ll 1$ in order for this component to be negligible at the beginning of BBN to ensure a radiation dominated era during $\mathrm{BBN}, T_{\mathrm{BBN}}=1 \mathrm{MeV}$ is the typical temperature of the Universe at the beginning of BBN, $\rho_{\text {rad }}$ is the radiation energy density and $4 \leq n_{D} \leq 8$ depending on the nature of the dark component ( $n_{D}=4$ radiation, $n_{D}=5$ non-relativistic matter, $n_{D}=6$ kination and $n_{D}>6$ can find justification in scalar decaying models).

The Friedmann equation reads

$$
H^{2}=\frac{8 \pi G}{3}\left(\rho_{B}+\rho_{D}\right)
$$

implying an expansion rate larger than in the standard case with important consequences for the stau-gravitino scenario. The Universe has a shorter reheating period as it cools down faster and therefore the gravitino production is less efficient. As for the stau, it is initially in thermal equilibrium and when the reactions between the stau and the plasma are not able to maintain equilibrium, the stau freezes out and then decays to the gravitino. With an expansion rate larger in this scenario, the freeze-out occurs earlier inducing a larger abundance of staus. The gravitino relic density from the stau decay is therefore larger as in the standard case. The dark component becomes negligible during $\mathrm{BBN}$ so its effect are extremely weak on the production of light elements.

\section{Gravitino dark matter}

\subsection{Thermal production}

The thermal production of gravitinos occurs during the reheating period after inflation. It is due to scattering processes emitting one gravitino. The gravitino yield is obtained by integrating the Boltzmann equation

$$
Y_{3 / 2}\left(T_{\mathrm{BBN}}\right)=-\int_{T_{R}}^{T_{\mathrm{BBN}}} d T \frac{C_{3 / 2}(T)}{s(T) H(T) T}
$$

where integration is performed between the reheating temperature $T_{R}$ and a low temperature taken as $T_{\mathrm{BBN}}, s(T)$ is the entropy, $H(T)$ is the Hubble parameter defined in eq. 2.2 and $C_{3 / 2}(T)$ is the collision term taking into account all scattering processes producing a gravitino. It has been 
calculated $[6,7,8]$ and we will take the result from [7]. In the standard cosmological scenario, Pradler and Steffen obtained the following gravitino yield

$$
Y_{\mathrm{PS}}\left(T_{\mathrm{BBN}}\right)=\sum_{\alpha=1}^{3}\left(1+\frac{M_{\alpha}^{2}}{3 m_{3 / 2}^{2}}\right) y_{\alpha} g_{\alpha}^{2} \ln \left(\frac{k_{\alpha}}{g_{\alpha}}\right)\left(\frac{T_{R}}{10^{10} \mathrm{GeV}}\right) .
$$

with $M_{\alpha}$ the gaugino mass, $g_{\alpha}$ the gauge couplings at $T_{R}, y_{\alpha}=\{0.65,1.6,4.3\} \times 10^{-12}$ and $k_{\alpha}=$ $\{1.266,1.313,1.271\}$. One can note that the gravitino production increases with the reheating temperature. Constraints on the dark matter relic puts an upper bound on the reheating temperature for a given gravitino mass.

In the non-standard scenario, we find a yield proportional to $Y_{\mathrm{PS}}\left(T_{\mathrm{BBN}}\right)$

$$
Y_{3 / 2}\left(T_{\mathrm{BBN}}\right)=Y_{\mathrm{PS}}\left(T_{\mathrm{BBN}}\right) \times{ }_{2} F_{1}\left(1 / N, 1 / 2 ; 1+1 / N ;-\kappa_{D}\left(\frac{T_{R}}{T_{\mathrm{BBN}}}\right)^{N}\right)
$$

where ${ }_{2} F_{1}$ is an hypergeometric function depending on $\kappa_{D}$ and $N=n_{D}-4$. For the given inputs, the hypergeometric function lies between 0 and 1 . This suppression on the gravitino production allows to increase the reheating temperature and still satisfy the dark matter relic constraint.

\subsection{Non-thermal production}

Assuming that each stau NLSP produces one gravitino, the non-thermal gravitino relic density can be related to the stau abundance:

$$
\Omega_{3 / 2}^{\mathrm{NTP}} h^{2}=\frac{m_{3 / 2}}{m_{\mathrm{NLSP}}} \Omega_{\mathrm{NLSP}} h^{2} .
$$

Since the stau abundance is higher because of an early freeze-out, the gravitino relic density also increases.

\subsection{Total dark matter}

The total gravitino relic density is the sum of the thermal and non-thermal production

$$
\Omega_{3 / 2} h^{2}=\Omega_{3 / 2}^{\mathrm{TP}} h^{2}+\Omega_{3 / 2}^{\mathrm{NTP}} h^{2} .
$$

In the non-standard scenario, the thermal term is smaller while the non-thermal is higher with respect to the standard cosmological case.

\section{Stau decay}

The stau mainly decays in a gravitino and a tau lepton with the following decay width

$$
\Gamma(\tilde{\tau} \rightarrow \tau \tilde{G})=\frac{1}{48 \pi} \frac{m_{\tilde{\tau}}^{5}}{M_{\mathrm{Pl}}^{2} m_{3 / 2}^{2}}\left(1-\frac{m_{3 / 2}^{2}}{m_{\tilde{\tau}}^{2}}\right)^{4} .
$$

The stau lifetime is approximately equal to $\tau \sim 1 / \Gamma(\tilde{\tau} \rightarrow \tau \tilde{G})$. Also, since the tau is unstable, its decay induces an electromagnetic cascade. The branching ratio and energy associated to this decay are

$$
B_{\mathrm{em}}=1-B_{\mathrm{had}} \simeq 1 \quad \text { and } \quad E_{\mathrm{em}}=\frac{1}{2}\left(\frac{m_{\mathrm{NLSP}}^{2}-m_{3 / 2}^{2}}{2 m_{\mathrm{NLSP}}}\right) .
$$


Hadronic decays (production of nucleons) are subdominant as they require a 4-body decay. Nevertheless, their impact on BBN can be strong and must be taken into account. The width decay of such processes are calculated as in [9]

$$
\Gamma\left(\tilde{\tau} \rightarrow \tau \tilde{G} q \bar{q} ; m_{q \bar{q}}\right)=\int_{m_{q \bar{q}}}^{m_{\tilde{\tau}}-m_{3 / 2}-m_{\tau}} d m_{q \bar{q}} \frac{d \Gamma(\tilde{\tau} \rightarrow \tau \tilde{G} q \bar{q})}{d m_{q \bar{q}}}
$$

with $m_{q \bar{q}}=2 \mathrm{GeV}$ is a cut-off mass below which no nucleons are produced. The energy associated to the hadronic decay is calculated in a similar way

$$
E_{\mathrm{had}}=\frac{1}{\Gamma(\tilde{\tau} \rightarrow \tau \tilde{G} q \bar{q})} \int_{m_{q \bar{q}}}^{m_{\tilde{\tau}}-m_{3 / 2}-m_{\tau}} d m_{q \bar{q}} m_{q \bar{q}} \frac{d \Gamma(\tilde{\tau} \rightarrow \tau \tilde{G} q \bar{q})}{d m_{q \bar{q}}}
$$

\section{Results}

We work in the CMSSM model with imputs: the gaugino mass $m_{1 / 2}$, the scalar mass $m_{0}$ and the trilinear coupling $A_{0}$ (taken equals to zero) at the GUT scale, $\tan \beta$ and the sign of $\mu$ (taken positive). We also take the gravitino mass as a free parameter. We use Suspect 2.41 to calculate the mass spectrum, micrOMEGAs 2.2 for the stau abundance, CalcHEP to calculate the width decays and energies and a private BBN code developed by Karsten Jedamzik for the light elements abundances produced during BBN.

We use the following constraints for the light elements derived from observations

$$
\begin{aligned}
1.2 \times 10^{-5} \leq \mathrm{D} / \mathrm{H} & \leq 5.3 \times 10^{-5} \\
{ }^{3} \mathrm{He} / \mathrm{D} & \leq 1.72 \\
Y_{p} & \leq 0.258 \\
8.5 \times 10^{-11} \leq{ }^{7} \mathrm{Li} / \mathrm{H} & \leq 2.5 \times 10^{-10} \\
0.015 \leq{ }^{6} \mathrm{Li} /{ }^{7} \mathrm{Li} & \leq 0.66
\end{aligned}
$$

The last two constraints are the lithium-7 and lithium- 6 abundances compatible with observations. In the following figures, SBBN values for lithium isotopes will be taken as

$$
\begin{aligned}
2.5 \times 10^{-10} \leq & { }^{7} \mathrm{Li} / \mathrm{H} \\
& { }^{6} \mathrm{Li} /{ }^{7} \mathrm{Li} \leq 0.015
\end{aligned}
$$

For the dark matter relic density, we use the abundance inferred from WMAP measurements [1]

$$
\Omega_{\mathrm{DM}} h^{2}=0.1099 \pm 0.0124
$$

As an element of comparison, Fig.1 presents the results for BBN and dark matter in the standard cosmological scenario. With fixed values of $m_{0}=80 \mathrm{GeV}$ and $\tan \beta=10$, one can solve both lithium problems and obtain the dark matter relic density for values of $m_{1 / 2} \sim 3-4 \mathrm{TeV}$ corresponding to stau masses of $m_{\tilde{\tau}} \sim 1.1-1.6 \mathrm{TeV}$ and reheating temperature $T_{R} \sim 10^{7} \mathrm{GeV}$.

Similar results are presented in Fig.2 for the non-standard cosmological scenario. Both figures have $\kappa_{D}=10^{-5}$ and $n_{D}=5$ (left) or $n_{D}=7$ (right). For $n_{D}=5$ the suppression of the gravitino 


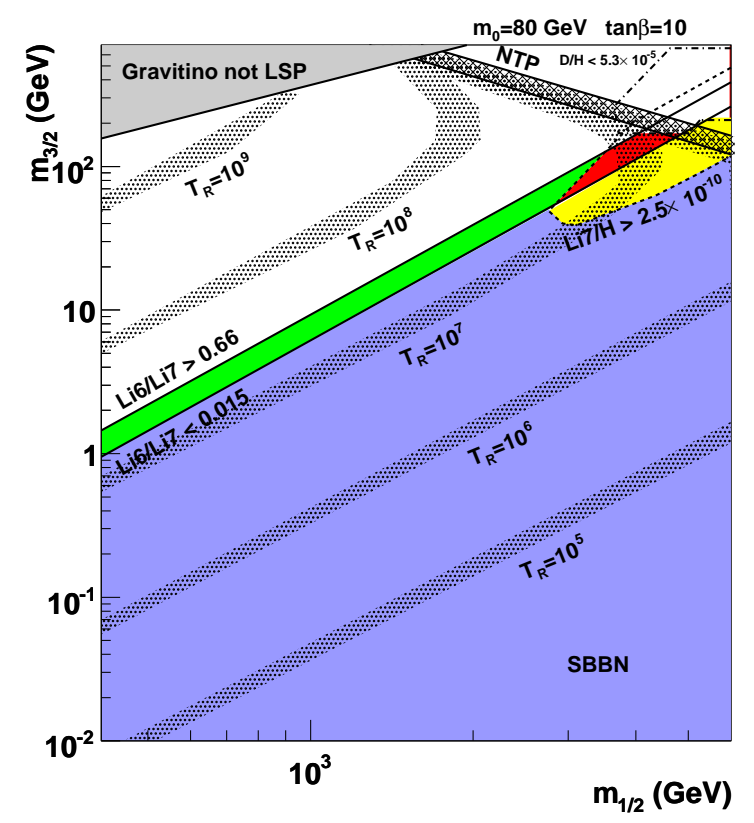

Figure 1: Big Bang Nucleosynthesis results in CMSSM with $m_{0}=80 \mathrm{GeV}$ and $\tan \beta=10$. The light blue region produces light elements abundances compatible with SBBN, masses solving the lithium-7 discrepancy (yellow), lithium-6 (green) and both problems (red). The hatched area labelled NTP indicates a gravitino relic density from non-thermal production alone compatible with measurements from WMAP and the other hatched correspond to the sum of TP+NTP for given reheating temperature.
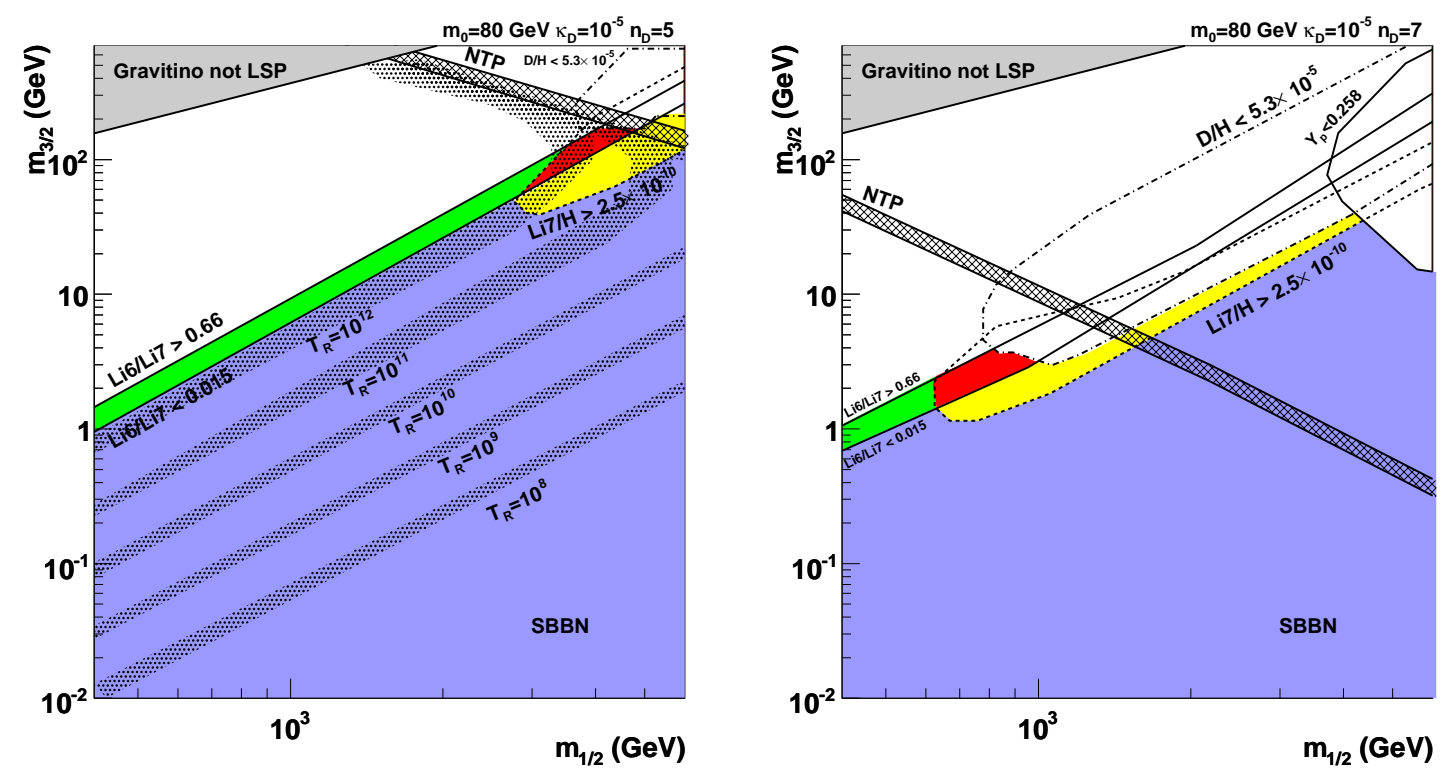

Figure 2: Results for Big Bang Nucleosynthesis in the modified expansion rate scenario with $\kappa_{D}=10^{-5}$ and $n_{D}=5$ (left) or $n_{D}=7$ (right). Colours and hatched areas defined as in previous figure. 
thermal production is strong enough to allow reheating temperature up to $T_{R} \sim 10^{12} \mathrm{GeV}$ but it does not affect much the stau abundance (less than one percent) and therefore the BBN results are very much similar to those obtained in the standard scenario. For $n_{D}=7$, the dark component is much larger in the early universe, the suppression of the gravitino thermal production is so strong that there is no constraint anymore on the reheating temperature. Also the stau abundance is affected and is enhanced. The consequence is that more decaying particles are present in the plasma during BBN and the impact on the light elements is stronger. In order to reproduce the right amount of lithium, lighter staus are required, shifting the regions of interest to smaller values of $m_{1 / 2}$ and smaller gravitino masses (to maintain the right lifetime for the staus). Notice that in this last scenario it is not possible to solve both lithium problems and obtain the right dark matter relic density. It is somehow possible to solve the lithium-7 problem and satisfy the dark matter relic density.

\section{Conclusion}

Supersymmetric models are a promicing extension of the Standard Model as they can address many issues in particle physics and cosmology. Here we focus on the dark matter candidate and the Big Bang Nucleosynthesis in the case of a stable gravitino LSP and a stau NLSP. In the standard cosmological scenario, in order to satisfy the BBN constraints and not overproduce dark matter, the reheating temperature upper bound is well below the temperature required for thermal leptogenesis and the supersymmetric particle must be quite heavy making production at LHC impossible.

The introduction of a dark component with a positive energy density suppresses the gravitino production during rehating allowing to have larger reheating temperatures in agreement with thermal leptogenesis. Also the abundance of stau is enhanced allowing to have lighter masses and still solve lithium problems and maybe reach LHC $(14 \mathrm{TeV})$ production capacity.

\section{Acknowledgements}

I would like to thank the organizers of IDM2010 and the warm welcome in Montpellier. This work was supported in part by the ANR project ToolsDMColl, BLAN07-2-194882.

\section{References}

[1] WMAP, E. Komatsu et al., (2008), arXiv:0803.0547 [astro-ph]

[2] S. Bailly, K. Jedamzik, and G. Moultaka, Phys. Rev. D80, 063509 (2009), 0812.0788

[3] S. Bailly, K.-Y. Choi, K. Jedamzik, and L. Roszkowski, JHEP 05, 103 (2009), 0903.3974

[4] A. Arbey and F. Mahmoudi, Phys. Lett. B669, 46 (2008), 0803.0741

[5] S. Bailly, arXiv:1008.2858 [hep-ph].

[6] M. Bolz, A. Brandenburg, and W. Buchmuller, Nucl. Phys. B606, 518 (2001), hep-ph/0012052

[7] J. Pradler and F. D. Steffen, Phys. Rev. D75, 023509 (2007), hep-ph/0608344

[8] V. S. Rychkov and A. Strumia, Phys. Rev. D75, 075011 (2007), hep-ph/0701104

[9] F. D. Steffen, JCAP 0609, 001 (2006), hep-ph/0605306 